\title{
DISTRIBUCIÓN DE LA ESCAMA BLANCA DEL MANGO AULACASPIS TUBERCULARIS NEWSTEAD (HEMIPTERA: DIASPIDIDAE) EN NAYARIT, MÉXICO
}

\section{Nadia Carolina GaRCíA-ÁlVAREZ, ${ }^{1}$ Mario Alfonso URÍAS-LÓPEZ, ${ }^{1 *}$ LuIS MARTÍN HERNÁNDEZ-FUENTES, ${ }^{1}$ JESÚS ASCENSIÓN GONZÁLEZ-CARRILLO, ${ }^{2}$ MARÍA HILdA PÉREZ-BARRAZA ${ }^{1}$ \& JORge Alberto OSUNA-GARCÍA ${ }^{\mathbf{1}}$}

${ }^{1}$ INIFAP. Campo Experimental Santiago Ixcuintla. Apdo. Postal 100, C. P. 63300, Santiago Ixcuintla, Nayarit.*Autor para correspondência: <urias.marioalfonso@inifap.gob.mx>.

${ }^{2}$ Colegio de Postgraduados. Carr. México-Texcoco km 36.5, Montecillo, 56230 Texcoco, Edo. México.

García-Álvarez, N. C., Urías-López, M. A., Hernández-Fuentes, L. M., González-Carrillo, J. A., Pérez-Barraza, M. H. \& Osuna-García, J. A. 2014. Distribución de la escama blanca del mango Aulacaspis tubercularis Newstead (Hemiptera: Diaspididae) en Nayarit, México. Acta Zoológica Mexicana (n. s.), 30(2): 321-336.

RESUMEN. El mango es el frutal más importante que se produce en Nayarit. Es atacado por la escama blanca Aulacaspis tubercularis Newstead (EBM), que puede producir pérdidas de hasta $50 \%$ de frutos cuando no se le controla. Debido a que se requiere un conocimiento de la presencia de la plaga para fines de manejo, el objetivo de este trabajo fue determinar la distribución geográfica e intensidad de infestación de la escama en Nayarit, con relación a diferentes alturas sobre el nivel del mar, a las dos principales variedades de mango y a la edad de los huertos. Durante tres años (2007-2009) se realizaron muestreos en los municipios productores de mango de Nayarit, en los periodos de fructificación (AbrilJunio de cada año). Durante 2007 se realizaron muestreos en 217 huertos comerciales, situados en 38 poblaciones; en 2008 se muestrearon 220 huertos de 45 poblados y en 2009 se muestrearon 83 huertos de 23 poblados. Se determinó que los huertos de mango situados en el sur del estado presentaron en general las mayores densidades de escama en follaje y las más altas infestaciones de frutos. Los municipios de Compostela y Bahía de Banderas resultaron con las mayores infestaciones de la plaga; San Blas y Tepic con infestaciones moderadamente altas; y Santiago Ixcuintla y Tecuala con infestaciones bajas. No se detectó la escama en Acaponeta, Rosamorada y Ruiz. Las mayores infestaciones de la EBM ocurrieron en huertos situados en alturas de 0-500 m, en huertos de mango 'Ataulfo' o en huertos menores de 10 años de edad.

Palabras clave: Distribución, infestación, mango ‘Ataulfo', mango 'Tommy Atkins'.

García-Álvarez, N. C., Urías-López, M. A., Hernández-Fuentes, L. M., González-Carrillo, J. A., Pérez-Barraza, M. H. \& Osuna-García, J. A. 2014. Distribution of the White mango scale 
Aulacaspis tubercularis Newstead (Hemiptera: Diaspididae) in Nayarit, Mexico. Acta Zoológica Mexicana (n. s.), 30(2): 321-336.

ABSTRACT. Mango is the main fruit produced in Nayarit, Mexico; it is damaged by the white mango scale (WMS) Aulacaspis tubercularis Newstead, which may produce losses of $50 \%$ if the pest is not controlled. Due to this problem, knowledge about its distribution in the State of Nayarit is required for management purposes. The aim of this study was to know the distribution of the scale in Nayarit, according to altitude, the two main cultivars and age of mango orchards. During three years (2007-2009) a program of sampling in all growing areas of Nayarit was conducted, during the periods of fructification and high densities of the pest (April-June of each year). During 2007, 217 mango orchards in 38 towns were sampled; 220 in 45 towns in 2008, and 83 mango orchards, in 23 towns in 2009 were sampled. It was found that mango orchards at the south of the state showed the higher densities of the scale on foliage, and the highest fruit infestation. Mango orchards from Compostela and Bahía de Banderas recorded the highest mango infestation; San Blas and Tepic resulted with moderate high infestations; Santiago Ixcuintla and Tecuala recorded low infestations. The pest was not detected in Acaponeta, Rosamorada and Ruiz. The highest WMS infestations were recorded in mango orchards at 0-500 of altitude, and also on 'Ataulfo' mango or in orchards less than 10 year old.

Key words: Distribution, infestation, ‘Ataulfo' mango, ‘Tommy Atkins’ mango.

\section{INTRODUCCIÓN}

El mango es el frutal más importante del estado de Nayarit; cuenta con una superficie establecida cercana a 22 mil hectáreas (SAGARPA-CESAVENAY 2007). Por mucho tiempo, las moscas de la fruta (Anastrepha spp.), han sido la principal plaga del cultivo en el estado, sin embargo, a partir de la última década le ha seguido en importancia la escama blanca del mango (EBM) Aulacaspis tubercularis Newstead. La escama puede reducir hasta en $50 \%$ el volumen comercial o calidad de la cosecha destinada para exportación (Urías 2006). La EBM se encuentra establecida en casi todas las zonas productoras de mango de Nayarit y se estima que infesta más de 10 mil hectáreas, principalmente en la zona costera del estado (Urías 2006; Urías-López et al. 2010).

Como ocurre con las demás especies de escamas de la familia Diaspididae (Triplehorn \& Johnson 2005), A tubercularis posee una armadura como protección del cuerpo. Esta es ovalada, plana y de color blanco opaco semitransparente. Las hembras después de nacer se dispersan en hojas y frutos donde forman una nueva colonia. Las colonias de escamas son solo machos de color blanco (Urías-López \& Flores-Canales 2005; Urías-López et al. 2010). En los machos la armadura es alargada y tiene dos depresiones (hendiduras) longitudinales paralelas al cuerpo. La EBM, además de infestar las hojas, en etapas de alta densidad, también invade tallos tiernos y frutos (Urías 2006; Urías-López et al. 2010), induce caída prematura del follaje (Wysoki et al. 1993; Urías-López et al. 2010), muerte de ramas (Wysoki et al. 1993) y es capaz de provocar hasta el 50\% de pérdidas en la cosecha (Urías 2006).

Aunque la EBM se encuentra ampliamente distribuida en las zonas productoras de mango del mundo, existe escasa información para fines de su manejo integrado, 
porque en pocos casos se ha manifestado como plaga. Respecto a la dinámica poblacional de la especie, se determinó que las densidades más altas ocurrieron de Octubre a Enero (Arias et al. 2004). En Nayarit, las poblaciones más bajas de la EBM ocurren durante el periodo de lluvias de verano (Julio-Noviembre), después se presenta una etapa de crecimiento poblacional (Diciembre-Febrero) y durante la época seca del año (Marzo-Junio) ocurren los niveles más altos de población (Urías-López \& FloresCanales 2005; González-Carrillo et al. 2008 a; Urías-López et al. 2010).

En el mismo estado de Nayarit se han registrado durante todo el año algunos depredadores de la EBM (González-Carrillo et al. 2008b), como el complejo de especies de "cargabasura", Ceraeochrysa sp. (Neuroptera: Chrysopidae), los coccinélidos Chilocorus cacti, Pentilia sp. y otro no identificado. Para el control químico de $A$. tubercularis, se recomiendan algunos productos comerciales (Arias et. al. 2004; Le Lagadec et al. 2006), no tradicionales como la citrolina (aceite mineral) y el uso de detergentes comerciales (Urías-López et al. 2013).

Debido a que la superficie infestada y la densidad de las poblaciones de la EBM se han incrementado en los últimos tiempos en Nayarit, se requiere tener mayor conocimiento de las zonas afectadas y del grado de infestación para fines de manejo. Por lo anterior el objetivo de este estudio fue determinar la distribución e intensidad de infestación de la plaga en Nayarit, con relación a las variedades principales de mango, a diferentes alturas sobre el nivel del mar y a la edad de los huertos.

\section{MATERIAL Y MÉTODOS}

Para determinar la distribución de la EBM en el estado, de 2007 a 2009 se muestrearon diferentes huertos comerciales de mango en las poblaciones de las principales zonas productoras del estado de Nayarit. Se realizó solo un muestreo por huerto durante el periodo de mayor abundancia poblacional de la escama, considerando el tiempo desde el cuajado ("amarre") de frutos hasta antes de la cosecha (Abril-Junio) de cada año. El estudio se realizó con las dos principales variedades de mango, 'Ataulfo' y 'Tommy Atkins'. Los muestreos se realizaron de acuerdo a la metodología sugerida por Urías et al (2010); se seleccionaron cinco árboles por huerto y en cada árbol se marcaron cuatro ramas orientadas a los puntos cardinales. De cada rama se seleccionaron cuatro hojas del penúltimo brote estacional de desarrollo (flujo vegetativo). Finalmente, en ambos lados de cada hoja, se hizo un conteo de hembras y colonias (machos) de la escama. También se consideró el porcentaje de frutos infestados.

Para determinar el porcentaje de frutos infestados, en todos los árboles se seleccionaron al azar cuatro brotes con presencia de frutos. En todos los frutos de cada brote seleccionado se determinó la presencia de hembras, y colonias de escamas. El cálculo del porcentaje de frutos infestados se realizó considerando aquellos con presencia de cualquier estado biológico y los frutos libres de la plaga. Como características de los 
huertos, se anotaron datos adicionales como la variedad, la altura sobre el nivel del mar y la edad del huerto (mayor o menor a 10 años).

Durante el primer año se muestrearon 217 huertos comerciales, situados en 38 localidades de los principales municipios productores de mango del estado. En San Blas, se muestrearon 94 huertos, en Tepic (62 huertos), en Compostela (46 huertos) y en Santiago Ixcuintla (15 huertos). En el segundo año se muestrearon 220 huertos comerciales de mango, situados en 45 poblados de los municipios de: Compostela (26 huertos), Tepic (40 huertos), San Blas (89 huertos), Santiago Ixcuintla (45 huertos), Tecuala (11 huertos), Ruiz (4 huertos) y Rosamorada (5 huertos). En 2009 se muestrearon 83 huertos comerciales en 23 localidades. En el tercer año solo se consideraron zonas poco o no examinadas en los años previos, principalmente de la zona Norte del estado y el municipio de Bahía de Banderas. La ubicación y número de huertos que se muestrearon en este caso fueron: 20 en Bahía de Banderas, 16 en Santiago Ixcuintla, Compostela, San Blas y Acaponeta, respectivamente, nueve en Tecuala, tres en Tepic y dos en Ruiz.

Análisis estadístico. Se realizaron análisis de varianza de las variables en estudio, mediante un diseño en bloques al azar con cinco repeticiones. Las medias se indicaron con su correspondiente error estándar ( \pm e e $)$, y para su comparación se utilizó la prueba de Tukey $(\mathrm{P} \leq 0.05)$. Todos los análisis se realizaron con el paquete estadístico SAS Institute (2000). Las variables fueron: hembras y colonias de escama (machos). De estas variables se generó una más denominada total de escamas que consistió en la suma de hembras y colonias.

\section{RESULTADOS Y DISCUSIÓN}

Distribución por localidades. En el primer año se detectaron diferencias en las poblaciones de EB. Los huertos de mango con las densidades poblacionales más altas fueron aquellos localizados en La Lima y Altavista, (municipio de Compostela), donde se registraron en promedio 3.16 y 2.31 colonias/hoja y un total (colonias más hembras) de 9.71 y 9.66 escamas/hoja, respectivamente (Cuadro 1). Poblaciones moderadamente altas se detectaron en huertos de los poblados El Capomo y San Isidro, donde las densidades de hembras+colonias fueron de más de una a menos de dos escamas/hoja en cada caso. Los huertos de poblaciones de Valle Morelos Gavilán Grande y Jesús Ma. Corte registraron las poblaciones más bajas, con valores no mayores a 0.05 hembras + colonias/hoja. Respecto a la escama en frutos, seis huertos presentaron una infestación superior al 40\% (Cuadro 1). Algunos huertos con alta infestación de escamas en follaje no necesariamente registraron la mayor infestación de frutos ( $v$.gr. Jolotemba y Las Cuatas), pero en general, huertos con bajas infestaciones de escama en follaje registraron bajas infestaciones de frutos (Cuadro 1). De las 38 localidades visitadas, solo las de Pozo de Villa, El Limón y Puerta de Mangos, del municipio de Santiago Ixcuintla, no registraron ninguna infestación. 
Cuadro 1. Variación en las densidades de $A$. tubercularis en hojas e índice de infestación de frutos de mango ( \pm e e) por localidades de Nayarit durante 2007.

\begin{tabular}{|c|c|c|c|c|}
\hline Localidad & Hembras/hoja & Colonias/hoja & Total/hoja & Frutos infestados $(\%)$ \\
\hline Jolotemba & $2.12(0.56) \mathrm{fgh}$ & $1.35(0.20) \mathrm{cd}$ & $3.47(0.69)$ efg & $65.8(6.1) \mathrm{a}$ \\
\hline Las Cuatas & $2.02(0.56) \mathrm{fgh}$ & $0.77(0.20)$ efgh & $2.80(0.69) \mathrm{fgh}$ & $62.5(6.1) \mathrm{ab}$ \\
\hline La Lima & $6.55(0.39) \mathrm{a}$ & $3.16(0.10) \mathrm{a}$ & $9.71(0.34) \mathrm{a}$ & 57.9 (4.3) abc \\
\hline Sta. Cruz & $4.47(0.25) b c$ & $1.50(0.09) \mathrm{c}$ & $5.97(0.31) b c$ & $51.6(2.7) \mathrm{bc}$ \\
\hline Altavista & $7.35(0.28) \mathrm{a}$ & $2.31(0.10) b$ & $9.66(0.34) \mathrm{a}$ & $49.3(3.0) \mathrm{c}$ \\
\hline El Divisadero & $5.05(0.23) b$ & $2.08(0.08) b$ & $7.14(0.28) b$ & $47.5(2.5) \mathrm{c}$ \\
\hline Ixtapa & $3.02(0.19) \mathrm{def}$ & $1.02(0.07) \mathrm{def}$ & $4.05(0.24) \mathrm{def}$ & $29.1(2.7) \mathrm{d}$ \\
\hline El Espino & $1.50(0.56)$ ghij & $0.35(0.20)$ hijk & 1.85 (0.69) hij & $28.8(6.1) \mathrm{d}$ \\
\hline Puga & $4.47(0.56) \mathrm{bc}$ & $0.90(0.20)$ efg & $5.37(0.69) \mathrm{cd}$ & $27.2(6.1) \mathrm{de}$ \\
\hline Las Varas & $3.39(0.19) \mathrm{cd}$ & $1.08(0.07) \mathrm{cdef}$ & $4.47(0.24) \mathrm{de}$ & $24.3(2.1) \mathrm{def}$ \\
\hline El Cora & $4.40(0.28) b c$ & $1.14(0.10)$ chef & $5.54(0.34) \mathrm{cd}$ & $23.8(3.0) \mathrm{def}$ \\
\hline Atonalisco & $4.50(0.39) b c$ & 0.97 (0.14) def & $5.47(0.49) \mathrm{cd}$ & $22.3(4.3) \mathrm{defg}$ \\
\hline Singayta & $2.28(0.28)$ defg & $0.75(0.10) \mathrm{fgh}$ & $3.04(0.34)$ efgh & 21.4 (3.0) defgh \\
\hline La Libertad & $3.35(0.18) \mathrm{cd}$ & $0.96(0.60)$ def & $4.31(0.23) \mathrm{def}$ & 20.1 (2.0) defghi \\
\hline El Capomo & $0.87(0.21) \mathrm{ijk}$ & $0.31(0.07) \mathrm{ijk}$ & $1.18(0.26) \mathrm{ijk}$ & 19.9 (2.3) defghi \\
\hline San Isidro & $2.16(0.25)$ efgh & $0.75(0.09) \mathrm{fgh}$ & $2.91(0.31) \mathrm{fgh}$ & 17.5 (2.7) defghij \\
\hline Tecuitata & $4.24(0.21) b c$ & $1.21(0.07) \mathrm{cde}$ & $5.46(0.26) \mathrm{cd}$ & 15.3 (2.3) efghijk \\
\hline Mesillas & $1.71(0.25) \mathrm{ghi}$ & $0.51(0.09)$ ghij & $2.22(0.31)$ ghi & 14.1 (2.7) fghijkl \\
\hline Jalco & $2.53(0.08)$ defg & $0.80(0.03)$ efg & $3.34(0.11)$ efg & 13.9 (1.0) fghijkl \\
\hline Las Palmas & $3.05(0.35)$ def & $1.22(0.13) \mathrm{cde}$ & $4.27(0.44) \mathrm{def}$ & 12.5 (2.7) fghijklm \\
\hline Otates & $0.40(0.56) \mathrm{jk}$ & $0.02(0.20) \mathrm{k}$ & $0.42(0.69) \mathrm{jk}$ & $10.0(6.1)$ ghijklm \\
\hline Mecatán & $2.68(0.25)$ defg & $0.71(0.09)$ fghi & $3.39(0.31)$ efg & 9.0 (2.7) hijklm \\
\hline El Ahuacate & $3.37(0.56) \mathrm{cd}$ & $0.87(0.20)$ efg & $4.25(0.69) \mathrm{def}$ & 7.9 (6.1) ijklm \\
\hline El Jicote & $1.00(0.19)$ hijk & $0.23(0.07) \mathrm{jk}$ & $1.23(0.24) \mathrm{ijk}$ & $6.6(2.2) \mathrm{jklm}$ \\
\hline Valle Lerma & $0.36(0.32) \mathrm{jk}$ & $0.00(0.11) \mathrm{k}$ & $0.36(0.40) \mathrm{jk}$ & 5.4 (3.6) jklm \\
\hline Huaristemba & $0.59(0.18) \mathrm{ijk}$ & $0.17(0.06) \mathrm{j} \mathrm{k}$ & $0.77(0.23) \mathrm{ijk}$ & $3.4(1.8) \operatorname{lmk}$ \\
\hline Navarrete & $0.63(0.14) \mathrm{ijk}$ & $0.17(0.05) \mathrm{j} \mathrm{k}$ & $0.80(0.17) \mathrm{ijk}$ & $2.9(1.5) \mathrm{lmk}$ \\
\hline Sauta & $0.56(0.23) \mathrm{ijk}$ & $0.14(0.08) \mathrm{j} \mathrm{k}$ & $0.70(0.28) \mathrm{jk}$ & $1.8(2.5) \mathrm{lm}$ \\
\hline 5 de Mayo & $0.33(0.15) \mathrm{jk}$ & $0.06(0.05) \mathrm{k}$ & $0.39(0.19) \mathrm{jk}$ & $1.8(1.7) \mathrm{lm}$ \\
\hline Est. Nanchi & $0.71(0.39) \mathrm{ijk}$ & $0.00(0.14) \mathrm{k}$ & $0.71(0.49) \mathrm{jk}$ & $1.5(4.3) \mathrm{lm}$ \\
\hline Jesús Ma. C. & $0.03(0.35) \mathrm{k}$ & $0.00(0.13) \mathrm{k}$ & $0.03(0.44) \mathrm{k}$ & $1.0(2.8) \mathrm{lm}$ \\
\hline Palomas & $0.25(0.79) \mathrm{k}$ & $0.00(0.29) \mathrm{k}$ & $0.25(0.98) \mathrm{k}$ & $0.2(6.1) \mathrm{m}$ \\
\hline Zalazares & $0.17(0.39) \mathrm{k}$ & $0.00(0.14) \mathrm{k}$ & $0.17(0.49) \mathrm{k}$ & $0.1(4.3) \mathrm{m}$ \\
\hline Valle Morelos & $0.05(0.32) \mathrm{k}$ & $0.00(0.11) \mathrm{k}$ & $0.05(0.40) \mathrm{k}$ & $0.1(3.5) \mathrm{m}$ \\
\hline Gav. Grande & $0.03(0.39) \mathrm{k}$ & $0.00(0.14) \mathrm{k}$ & $0.03(0.49) \mathrm{k}$ & $0.1(4.3) \mathrm{m}$ \\
\hline
\end{tabular}

Valores por columna con la misma letra son estadísticamente iguales (Tukey, $p \leq 0.05$ ).

Los huertos de tres localidades no registraron infestación de escamas. 
En el siguiente año se detectaron diferencias significativas entre las localidades con relación a las variables evaluadas (Cuadro 2). Los huertos de El Divisadero y Santa Cruz presentaron valores promedio superiores a dos hembras/hoja. En el caso de colonias, el promedio fue bajo en todas las localidades con valores inferiores a 0.2 colonias/hoja. El total (hembras+colonias) fue en promedio de 5.3, 2.3 y 1.3/hoja,

Cuadro 2. Variación en las densidades de $A$. tubercularis en hojas e índice de infestación de frutos de mango ( \pm e e) por localidades de Nayarit durante 2008.

\begin{tabular}{|c|c|c|c|c|}
\hline Localidad & Hembras/hoja & Colonias/hoja & Total/hoja & Frutos infestados $(\%)$ \\
\hline El Divisadero & $5.10(0.13) \mathrm{a}$ & $0.20(0.02) \mathrm{a}$ & $5.30(0.1) \mathrm{a}$ & $61.0(2.0) \mathrm{a}$ \\
\hline Santa Cruz & $2.10(0.20) b$ & $0.20(0.03) \mathrm{ab}$ & $2.30(0.20) b$ & $43.0(3.0) \mathrm{b}$ \\
\hline Altavista & $1.00(0.20) \mathrm{cd}$ & $0.10(0.03) \mathrm{bcd}$ & $1.10(0.20) \mathrm{cd}$ & $21.0(3.0) \mathrm{c}$ \\
\hline Sauta & $1.00(0.12) \mathrm{cde}$ & $0.10(0.02) \mathrm{cd}$ & $1.10(0.13)$ cde & $19.0(2.0) \mathrm{c}$ \\
\hline Coloradas & $1.22(0.13) \mathrm{c}$ & $0.10(0.02) \mathrm{cd}$ & $1.32(0.14) \mathrm{c}$ & $17.5(2.0) \mathrm{cd}$ \\
\hline El Ahuacate & $1.00(0.30) \mathrm{cde}$ & $0.00(0.04) \mathrm{d}$ & $1.00(0.30)$ cdef & $17.0(4.0)$ cde \\
\hline El Cora & $1.00(0.12) \mathrm{def}$ & $0.13(0.02) a b c$ & $1.00(0.13)$ cdefg & $16.5(2.0) \mathrm{cde}$ \\
\hline Las Palmas & $0.50(0.21) \mathrm{def}$ & $0.05(0.03) \mathrm{cd}$ & $0.53(0.22)$ defgh & $16.0(3.2) \mathrm{cdef}$ \\
\hline La Libertad & $1.00(0.10) \mathrm{def}$ & $0.03(0.01) \mathrm{d}$ & $1.03(0.10)$ defgh & $16.0(1.2) \mathrm{cdef}$ \\
\hline Tecuitata & $0.40(0.13) \mathrm{def}$ & $0.02(0.02) \mathrm{d}$ & $0.42(0.14)$ defgh & $14.3(2.0)$ cdefg \\
\hline Atonalisco & $0.50(0.10) \mathrm{def}$ & $0.10(0.01) \mathrm{cd}$ & $0.60(0.10)$ defgh & 14.0 (1.4) cdefgh \\
\hline El Llano & $0.50(0.15) \mathrm{def}$ & $0.10(0.02) \mathrm{cd}$ & $0.60(0.20)$ defgh & 13.4 (2.2) cdefgh \\
\hline Mecatán & $0.30(0.10)$ ef & $0.01(0.02) \mathrm{d}$ & $0.31(0.10)$ efgh & 9.1 (1.4) defghi \\
\hline Huaristemba & $0.40(0.10) \mathrm{def}$ & $0.10(0.02) \mathrm{cd}$ & $0.50(0.10)$ defgh & $8.0(1.5)$ efghi \\
\hline Ixtapa & $0.30(0.12)$ ef & $0.03(0.02) \mathrm{d}$ & $0.33(0.13)$ efgh & 7.1 (2.0) fghi \\
\hline El capomo & $0.30(0.12)$ ef & $0.03(0.02) \mathrm{d}$ & $0.33(0.13)$ efgh & 7.0 (2.0) ghi \\
\hline C. de mayo & $0.30(0.10)$ ef & $0.04(0.02) \mathrm{d}$ & $0.32(0.10)$ efgh & 6.0 (1.4) ghi \\
\hline San Isidro & $0.13(0.21)$ ef & $0.03(0.03) \mathrm{d}$ & $0.16(0.22)$ efgh & $5.0(3.2) \mathrm{hi}$ \\
\hline Jalco & $0.10(0.10) \mathrm{f}$ & $0.01(0.01) \mathrm{d}$ & $0.11(0.10) \mathrm{h}$ & $2.5(1.0) \mathrm{i}$ \\
\hline J. Ma. Corte & $0.04(0.15) \mathrm{f}$ & $0.01(0.02) \mathrm{d}$ & $0.05(0.20) \mathrm{h}$ & $2.4(2.2) \mathrm{i}$ \\
\hline Mazatán & $0.15(0.15)$ ef & $0.00(0.02) \mathrm{d}$ & $0.15(0.20)$ efgh & $2.0(2.2) \mathrm{i}$ \\
\hline El Jicote & $0.10(0.12) \mathrm{f}$ & $0.00(0.02) \mathrm{d}$ & $0.10(0.13) \mathrm{gh}$ & $2.0(2.0) \mathrm{i}$ \\
\hline Salazares & $0.10(0.13) \mathrm{f}$ & $0.03(0.02) \mathrm{d}$ & $0.13(0.14)$ fgh & $2.0(2.0) \mathrm{i}$ \\
\hline Guinea & $0.04(0.15) \mathrm{f}$ & $0.00(0.02) \mathrm{d}$ & $0.04(0.20) \mathrm{h}$ & $2.0(2.3) \mathrm{i}$ \\
\hline Navarrete & $0.03(0.10) \mathrm{f}$ & $0.00(0.02) \mathrm{d}$ & $0.03(0.10) \mathrm{h}$ & $1.0(2.0) \mathrm{i}$ \\
\hline Singayta & $0.03(0.20) \mathrm{f}$ & $0.00(0.03) \mathrm{d}$ & $0.03(0.20) \mathrm{h}$ & $1.0(3.0) \mathrm{i}$ \\
\hline El Limón & $0.01(0.10) \mathrm{f}$ & $0.00(0.01) \mathrm{d}$ & $0.01(0.10) \mathrm{h}$ & $0.2(1.2) \mathrm{i}$ \\
\hline
\end{tabular}

Valores por columna con la misma letra son estadísticamente iguales (Tukey, $p \leq 0.05$ ).

Los huertos de 18 localidades no registraron infestación de escamas. 
respectivamente. Las localidades Altavista, El Divisadero y Santa Cruz, repitieron respecto al primer año como algunas de las localidades con alta infestación de huertos. También se detectaron diferencias significativas en el porcentaje de frutos infestados. Nueve localidades promediaron por arriba del 15\% de infestación de frutos, que en general corresponden a las de mayor infestación de escama en follaje. Entre estas, las que presentaron los porcentajes más altos fueron El Divisadero (61\%), Santa Cruz (43\%), Altavista (21\%) y Sauta (19\%) (Cuadro 2). En las siguientes localidades no se detectó infestación de escamas: La Yerba, Gavilán Grande, Las Parejas, La Mojonera, El Mil, El Vado, Pozo de Villa, Gavilán Chico, Las Minitas, San Dieguito, Emiliano Zapata, Chilapa, Estación Nanchi, Sentispac, La Redención, Puerta Colorada, Valle Lerma y Valle Morelos. Estas localidades correspondieron a los municipios de Ruiz, Rosamorada, Santiago Ixcuintla y Tecuala.

En el tercer año también se detectaron diferencias significativas entre localidades respecto a las variables estudiadas. Los promedios más altos de hembras se registraron en huertos de los poblados el Tonino, La Peñita, La Florida y el Monteón, encontrándose valores de 7.55 a 10.33 hembras/hoja (Cuadro 3). Los huertos de El

Cuadro 3. Variación en las densidades de A. tubercularis en hojas e índice de infestación de frutos de mango ( \pm e e) por localidades de Nayarit durante 2009.

\begin{tabular}{lllll}
\hline Localidad & Hembras/hoja & Colonias/hoja & Total/hoja & Frutos infestados (\%) \\
\hline El Monteón & $10.33(0.33) \mathrm{a}$ & $1.68(0.08) \mathrm{ab}$ & $12.01(0.31) \mathrm{a}$ & $42.4(3.3) \mathrm{bc}$ \\
La Florida & $8.92(0.57) \mathrm{abc}$ & $2.00(0.16) \mathrm{a}$ & $10.92(0.64) \mathrm{ab}$ & $10.4(3.0) \mathrm{d}$ \\
La Peñita & $9.19(0.33) \mathrm{ab}$ & $1.59(0.03) \mathrm{ab}$ & $10.78(0.37) \mathrm{ab}$ & $50.6(3.8) \mathrm{b}$ \\
El Tonino & $7.55(0.28) \mathrm{abc}$ & $1.32(0.08) \mathrm{bc}$ & $8.88(0.32) \mathrm{bc}$ & $45.1(3.3) \mathrm{bc}$ \\
San Juan & $7.11(0.20) \mathrm{dc}$ & $0.55(0.06) \mathrm{efg}$ & $7.66(0.22) \mathrm{dc}$ & $75.9(2.3) \mathrm{a}$ \\
Sayulita & $5.45(0.57) \mathrm{de}$ & $0.95(0.16) \mathrm{de}$ & $6.40(0.64) \mathrm{de}$ & $78.1(6.6) \mathrm{a}$ \\
Reforma Agr. & $4.64(0.26) \mathrm{ef}$ & $1.61(0.07) \mathrm{ab}$ & $6.24(0.28) \mathrm{def}$ & $19.8(3.0) \mathrm{cd}$ \\
Mezcales & $4.79(0.22) \mathrm{ef}$ & $0.48(0.06) \mathrm{efg}$ & $5.26(0.24) \mathrm{ef}$ & $83.3(2.5) \mathrm{a}$ \\
Las Palmas & $3.28(0.26) \mathrm{fg}$ & $1.29(0.07) \mathrm{bc}$ & $4.57(0.28) \mathrm{efg}$ & $43.5(3.0) \mathrm{bc}$ \\
Mecatán & $3.00(0.57) \mathrm{fg}$ & $1.15(0.16) \mathrm{de}$ & $4.12(0.64) \mathrm{efg}$ & $45.0(6.6) \mathrm{bc}$ \\
Chula Vista & $3.32(0.57) \mathrm{fg}$ & $0.72(0.16) \mathrm{de}$ & $4.05(0.64) \mathrm{fg}$ & $39.2(6.6) \mathrm{bc}$ \\
Las Lomas & $2.47(0.40) \mathrm{g}$ & $0.51(0.11) \mathrm{efg}$ & $2.99(0.45) \mathrm{gh}$ & $35.6(4.7) \mathrm{bc}$ \\
Salazares & $2.29(0.10) \mathrm{gh}$ & $0.26(0.11) \mathrm{fg}$ & $2.55(0.45) \mathrm{ghi}$ & - \\
El Jicote & $1.38(0.57) \mathrm{gh}$ & $0.20(0.16) \mathrm{fg}$ & $1.58(0.64) \mathrm{hij}$ & - \\
Est. Nanchi & $0.36(0.41) \mathrm{hi}$ & $0.08(0.12) \mathrm{g}$ & $0.44(0.46) \mathrm{hij}$ & $100.0(29.3) \mathrm{a}$ \\
Mojarritas & $0.26(0.12) \mathrm{i}$ & $0.08(0.07) \mathrm{g}$ & $0.34(0.28) \mathrm{ij}$ & $100.0(4.6) \mathrm{a}$ \\
\hline
\end{tabular}

Valores por columna con la misma letra son estadísticamente iguales (Tukey, $p \leq 0.05$ ).

Los huertos de 6 localidades no registraron infestación de escamas. 
Jicote, Estación Nanchi y Mojarritas, registraron promedios bajos de 1.38, $0.36 \mathrm{y}$ 0.26 hembras/hoja, respectivamente. Con relación al número de colonias, los promedios más altos, superiores a 1.5 de colonias/hoja, se presentaron en los huertos de La Peñita, Reforma Agraria, El Monteón y La Florida. En cuanto al total de escamas (hembras+colonias), los registros más altos ocurrieron en El Monteón (12.01) La Florida (12.92), La Peñita (10.78) y El Tonino (8.88) escamas/hoja (Cuadro 3). Respecto al porcentaje de frutos infestados, aunque Estación Nanchi y Mojarritas tuvieron una baja población de escamas, fueron las que registraron 100\% de infestación de frutos. Otros huertos con alta infestación de frutos fueron en Mezcales (83.3), San Juan (75.9) y Sayulita (78.1) (Cuadro 3). Las Lomas y Chula Vista se registraron valores medios de infestación de 35.6 a 39.2\%. En Acaponeta, Redención, Ruiz, Parejas, Tecuala, y Yago, no se detectó infestación de la EBM en hojas ni en frutos.

Distribución por municipio. En 2007, se encontraron diferencias significativas entre los municipios productores de mango estudiados. En los huertos de Compostela se registraron las densidades de población más altas, con 3.23 hembras/hoja y 1.15 colonias por hoja, en promedio. En esta misma localidad, el total (hembras+colonias) fue también bastante alto (4.39/hoja), comparado con el resto de los municipios (Cuadro 4). Otros municipios con un grado importante de infestación fueron San Blas y Tepic con 3.16 y 1.13 escamas/hoja, respectivamente. Los huertos de Santiago Ixcuintla presentaron las densidades más bajas al promediar 0.23 hembras/hoja, 0.03 colonias/ hoja y un total (hembras+colonias) de 0.27 escamas/hoja (Cuadro 4). Por otra parte, se detectaron diferencias significativas en el porcentaje de frutos infestados entre municipios. En Compostela se encontró la mayor infestación de frutos (29.7\%), seguido de San Blas (14.6\%), Tepic (4.7\%) y Santiago Ixcuintla (1.1\% (Fig. 1).

En 2008, también se detectaron diferencias significativas entre municipios. El número más alto de hembras/hoja se registró en Compostela (4.0), seguido de Tepic (3.2) y San Blas (3.01) (Cuadro 4). El promedio más bajo se registró en Tecuala (0.02), mientras que en Ruiz y Rosamorada no se encontraron hembras. De igual manera, la mayor cantidad de colonias/hoja correspondió a Compostela (1.6), el número más bajo se registró en Tecuala (0.01) y no se detectaron colonias en Ruiz y Rosamorada. Con relación al total de escamas (hembras+colonias), la mayor densidad se manifestó en los huertos de Compostela con 5.5, seguidos por los de Tepic (5.0) y San Blas (4.24). El promedio más bajo de total de escamas se observó en Tecuala (0.03). Con respecto al porcentaje de frutos infestados, también ocurrieron diferencias entre municipios, siendo Compostela el que registró la mayor infestación en frutos $(18.2 \%)$ y Tecuala el más bajo (0.3\%) (Fig. 2).

En 2009, una vez más se detectaron diferencias significativas entre los municipios visitados. En esta ocasión, los valores más altos correspondieron a los huertos de Bahía de Banderas, con 2.7 hembras y 2.76 total de escamas/hoja, respectivamente (Cuadro 4). Por el contrario, Santiago Ixcuintla registró el promedio más bajo con 
Cuadro 4. Densidad poblacional de A. tubercularis en hojas de mango ( \pm e e) en municipios de Nayarit durante tres años.

\begin{tabular}{|c|c|c|c|}
\hline Municipio & Hembras/hoja & Colonias/hoja & Total/hoja \\
\hline \multicolumn{4}{|c|}{2007} \\
\hline Compostela & $3.23(0.08) \mathrm{a}$ & $1.15(0.03) \mathrm{a}$ & $4.39(0.10) \mathrm{a}$ \\
\hline San Blas & $2.42(0.05) b$ & $0.74(0.02) b$ & $3.16(0.07) b$ \\
\hline Tepic & $0.93(0.10) \mathrm{c}$ & $0.19(0.03) \mathrm{c}$ & $1.13(0.13) \mathrm{c}$ \\
\hline Santiago Ixc. & $0.23(0.11) \mathrm{d}$ & $0.03(0.04) \mathrm{d}$ & $0.27(0.14) \mathrm{d}$ \\
\hline \multicolumn{4}{|c|}{2008} \\
\hline Compostela & $4.00(0.12) \mathrm{a}$ & $1.60(0.04) \mathrm{a}$ & $5.50(0.15) \mathrm{a}$ \\
\hline Tepic & $3.20(0.09) \mathrm{b}$ & $0.90(0.03) \mathrm{c}$ & $5.00(0.12) b$ \\
\hline San Blas & $3.01(0.06) b$ & $1.23(0.02) \mathrm{b}$ & $4.24(0.10) b$ \\
\hline Santiago Ixc. & $0.58(0.10) \mathrm{c}$ & $0.28(0.03) \mathrm{d}$ & $0.86(0.11) \mathrm{c}$ \\
\hline Tecula & $0.02(0.20) \mathrm{c}$ & $0.01(0.10) \mathrm{e}$ & $0.03(0.22) \mathrm{d}$ \\
\hline Acaponeta & $0.00(0.09) \mathrm{d}$ & $0.00(0.01) \mathrm{e}$ & $0.00(0.05) \mathrm{d}$ \\
\hline Ruiz & $0.00(0.30) \mathrm{c}$ & $0.00(0.11) \mathrm{e}$ & $0.00(0.40) \mathrm{d}$ \\
\hline Rosamorada & $0.00(0.25) \mathrm{c}$ & $0.00(0.10) \mathrm{e}$ & $0.00(0.30) \mathrm{d}$ \\
\hline \multicolumn{4}{|c|}{2009} \\
\hline Bahía de B. & $2.7(0.1) \mathrm{a}$ & $0.06(0.01) \mathrm{a}$ & $2.76(0.08) \mathrm{a}$ \\
\hline Compostela & $1.0(0.1) b$ & $0.05(0.02) \mathrm{a}$ & $1.02(0.10) b$ \\
\hline San Blas & $0.7(0.1) b$ & $0.04(0.02) \mathrm{ab}$ & $0.77(0.11) b$ \\
\hline Santiago Ixc. & $0.0(0.1) \mathrm{c}$ & $0.01(0.02) b c$ & $0.01(0.10) \mathrm{c}$ \\
\hline Acaponeta & $0.0(0.1) \mathrm{c}$ & $0.0(0.02) \mathrm{c}$ & $0.00(0.10) \mathrm{c}$ \\
\hline Tecuala & $0.0(0.2) \mathrm{c}$ & $0.0(0.02) \mathrm{c}$ & $0.00(0.12) \mathrm{c}$ \\
\hline Ruiz & $0.0(0.1) \mathrm{c}$ & $0.0(0.05) \mathrm{c}$ & $0.00(0.25) \mathrm{c}$ \\
\hline
\end{tabular}

Valores por columna y año con la misma letra son estadísticamente iguales (Tukey, $p \leq 0.05$ ).

apenas 0.01 total/hoja (hembras+colonias). En general, en ese año se apreciaron niveles bajos de poblaciones de escamas en el follaje, sin embargo, los porcentajes de infestación en los frutos fueron altos (Fig. 3). En el municipio de Bahia de Banderas se observó la infestación más alta de frutos $(69.4 \%)$ y la más baja en Santiago Ixcuintla $(19.3 \%)$. Durante este estudio no se presentaron infestaciones de escama en follaje ni en frutos, en los huertos de Acaponeta, Rosamorada y Ruiz (Cuadro 4).

Los resultados muestran que la distribución de la EBM es mayor en municipios del sur del Estado, como Compostela, Bahía de Bandera e incluso San Blas. Estos son congruentes si se considera que en Compostela se originó el problema y a que la mayoría de los huertos de esos municipios se encuentran a alturas más cercanas al nivel 


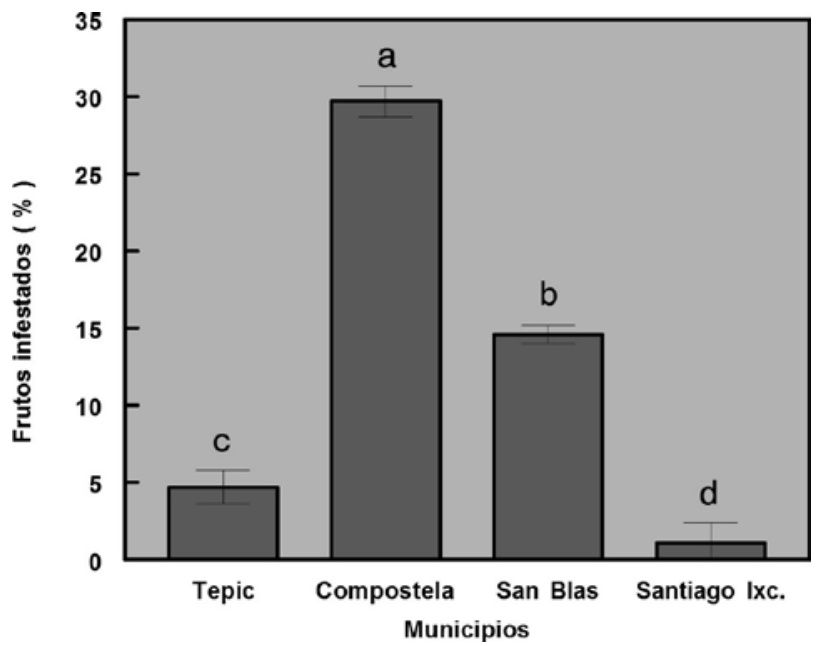

Figura 1. Índice de infestación de frutos de mango por $A$. tubercularis en municipios de Nayarit en 2007.

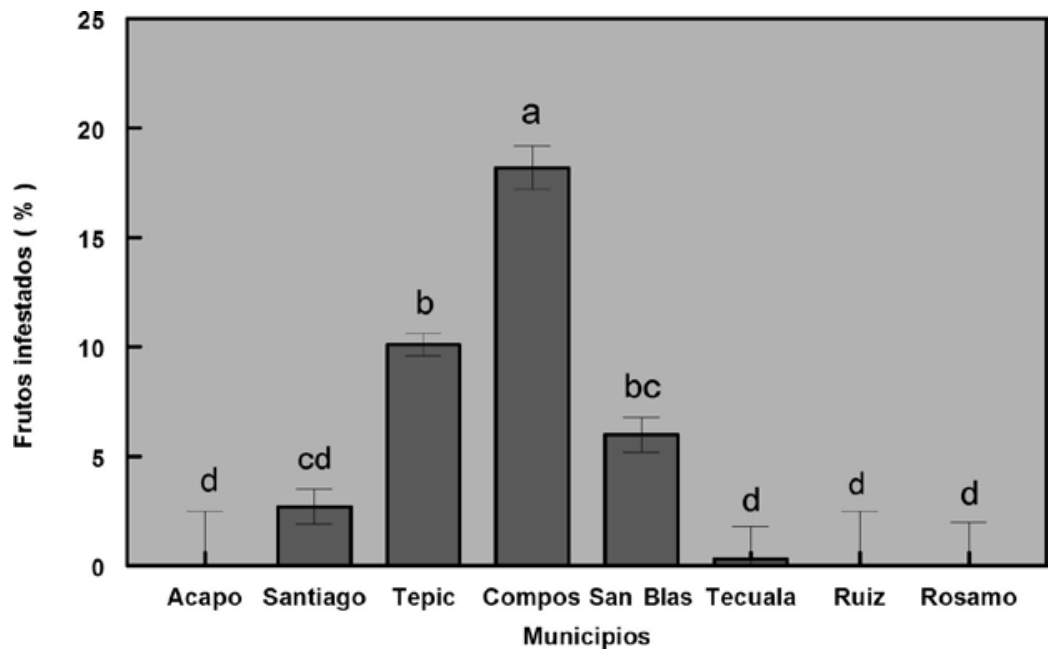

Figura 2. Índice de infestación de frutos de mango por A. tubercularis en municipios de Nayarit en 2008.

del mar. Esta característica de los huertos resultaría más favorable para el desarrollo de la plaga, al haber temperaturas más altas que en huertos de municipios situados a mayor altura. Este es el caso de Tepic, donde se localizaron huertos en mediana a gran altitud, entre 400 y $800 \mathrm{~m}$ sobre el nivel del mar. Con base en este argumento, 


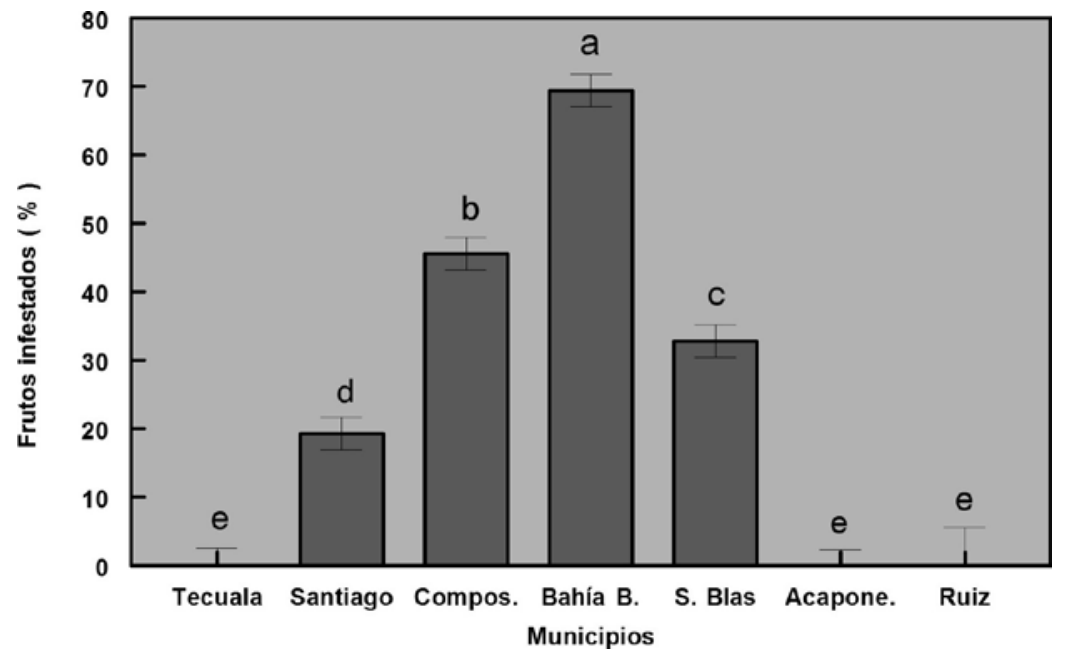

Figura 3. Índice de infestación de frutos de mango por A. tubercularis en municipios de Nayarit en 2009.

en Santiago Ixcuintla se esperarían altas infestaciones de escamas al estar los huertos cercanos al nivel del mar. Sin embargo, aquí la introducción de EBM es más reciente que en el resto de los municipios, y por tanto muy pocos huertos están aún infestados, lo que genera promedios bajos de infestación por municipio.

Distribución por alturas. Durante el periodo de muestreo, se detectaron diferencias significativas entre huertos situados a diferentes alturas sobre el nivel del mar. En el primero de ellos (2007), las poblaciones fueron mayores en huertos situados por arriba de los $600 \mathrm{msnm}$, con 2.8 hembras, 0.91 colonias y 3.71 total/hoja (Cuadro 5). Contrario a lo esperado, el promedio más bajo se presentó en huertos cercanos al nivel del mar (no más de $149 \mathrm{msnm}$ ), con 2.07 hembras, 0.71 colonias y 2.78 total/hoja. Por otra parte, la infestación de frutos también varió a diferentes alturas. El registro más alto se localizó entre $0-149$ msnm (18.3\%) y el más bajo (11\%), por arriba de los $600 \mathrm{msnm}$ (Fig. 4).

Durante el segundo año (2008), la densidad más alta de EBM se detectó en huertos situados entre 300-449 msnm, con un promedio de 4.53 hembras por hoja, mientras que el promedio más bajo se encontró en huertos situados por arriba de los $600 \mathrm{msnm}$ (2.78 hembras/hoja) (Cuadro 5). De acuerdo al total de escamas (hembras más colonias), las poblaciones de escamas con mayor densidad fueron aquellos localizados entre 300-449 msnm, seguido de los situados a alturas entre 150-299 msnm, mientras que los más bajos, en los huertos ubicados por arriba de $600 \mathrm{msnm}$. Por otra parte, el porcentaje de frutos infestados (15.5) fue mayor en huertos del intervalo 300-449 m y el más bajo (0.2) en los de más de $600 \mathrm{~m}$ de altura (Fig. 5). 
García-Álvarez et al.: Distribución de la escama blanca del mango en Nayarit

Cuadro 5. Variación en la densidad de poblaciones de $A$. tubercularis ( \pm e e) en follaje de mango por altura sobre el nivel del mar de Nayarit.

\begin{tabular}{lccl}
\hline Altura $(\mathrm{m})$ & Hembras/hoja & Colonias/hoja & Total/hoja \\
\hline & & 2007 & \\
$0-149$ & $2.07(0.06) \mathrm{b}$ & $0.71(0.02) \mathrm{b}$ & $2.78(0.08) \mathrm{c}$ \\
$150-299$ & $2.84(0.13) \mathrm{a}$ & $0.80(0.04) \mathrm{ab}$ & $3.64(0.16) \mathrm{ab}$ \\
$300-449$ & $2.47(0.17) \mathrm{a}$ & $0.69(0.06) \mathrm{b}$ & $3.16(0.22) \mathrm{bc}$ \\
$450-600$ & $2.82(0.12) \mathrm{a}$ & $0.84(0.05) \mathrm{ab}$ & $3.67(0.15) \mathrm{ab}$ \\
$>600$ & $2.80(0.18) \mathrm{a}$ & $0.91(0.07) \mathrm{a}$ & $3.71(0.23) \mathrm{a}$ \\
& & 2008 & \\
$0-149$ & $2.88(0.08) \mathrm{c}$ & $1.13(0.03) \mathrm{c}$ & $4.02(0.10) \mathrm{c}$ \\
$150-299$ & $3.46(0.12) \mathrm{b}$ & $1.34(0.04) \mathrm{ab}$ & $5.00(0.14) \mathrm{b}$ \\
$300-449$ & $4.53(0.21) \mathrm{a}$ & $1.39(0.08) \mathrm{a}$ & $5.92(0.26) \mathrm{a}$ \\
$450-600$ & $3.45(0.15) \mathrm{b}$ & $1.12(0.05) \mathrm{c}$ & $4.56(0.19) \mathrm{bc}$ \\
$>600$ & $2.78(0.23) \mathrm{c}$ & $1.18(0.08) \mathrm{bc}$ & $3.96(0.28) \mathrm{c}$ \\
& & 2009 & $3.7(0.1) \mathrm{a}$ \\
$0-199$ & $3.2(0.1) \mathrm{a}$ & $0.6(0.1) \mathrm{b}$ & $4.2(0.9) \mathrm{b}$ \\
\hline
\end{tabular}

Valores por columna y año con la misma letra son estadísticamente iguales (Tukey, $p \leq 0.05$ ).

En el último muestreo (2009), solamente se consideraron dos intervalos de altura. De éstos, el de 400-599 msnm, registró el mayor promedio de colonias/hoja (1.6) y total de hembras + colonias/hoja (4.2), comparado con el intervalo de 0-199 msnm (Cuadro 5). En cambio, la mayor infestación de frutos se observó en el intervalo cercano al nivel del mar (0-199), con un promedio de 53.8\% de frutos infestados contra $45 \%$ en huertos de $400-599$ de altura.

De acuerdo con la información obtenida, se esperaría una mayor población de escamas en huertos cercanos al nivel del mar, debido a la acumulación de calor durante el año, sin embargo debe tomarse en cuenta que en Santiago Ixcuintla aún no se había establecido totalmente la plaga, lo que debió haber influido notoriamente en el bajo promedio. Por otra parte, debe considerarse que este es resultado del muestreo durante solo una parte del año, en un periodo de alta población de escamas, por lo que podría cambiar si se consideran las poblaciones durante todo el año.

Distribución por cultivar. En el primer periodo de estudio, se encontró que en la variedad 'Ataulfo' hubo un poco menos del doble de escamas que en 'Tommy Atkins', al igual que una mayor infestación de frutos (16.1\%) (Cuadro 6). Para el segundo periodo, los promedios de hembras, colonias y el total de escamas, volvieron a ser más altos en 'Ataulfo' que en 'Tommy Atkins'. El porcentaje de frutos infestados por la EBM fue igualmente más alto en 'Ataulfo' (8.6\%) que en 'Tommy Atkins' (6.1). 


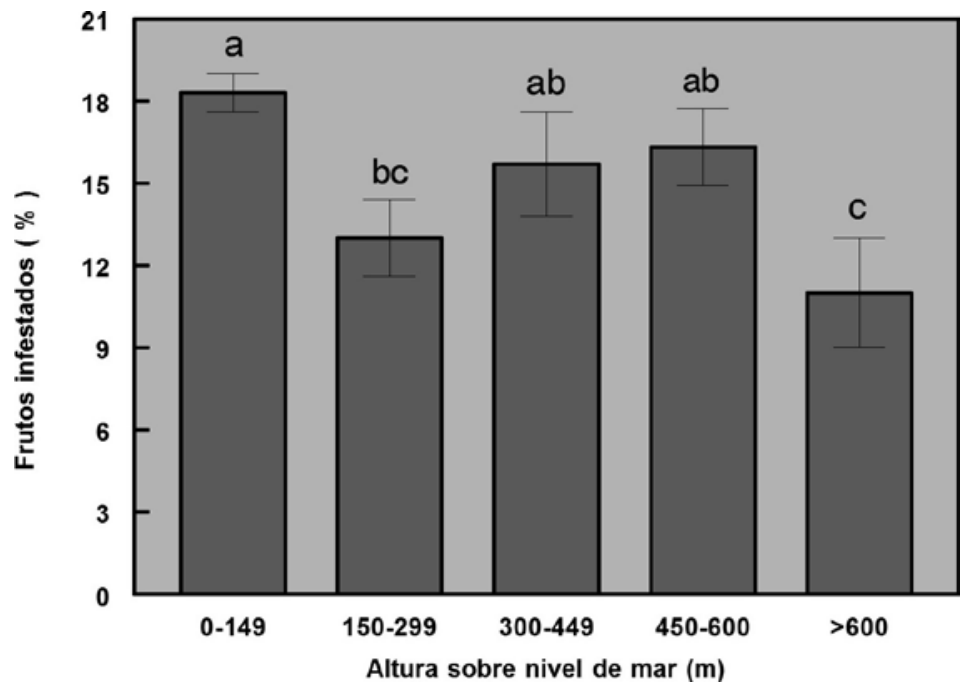

Figura 4. Infestación de frutos de mango por A. tubercularis a diferentes alturas de Nayarit en 2007.

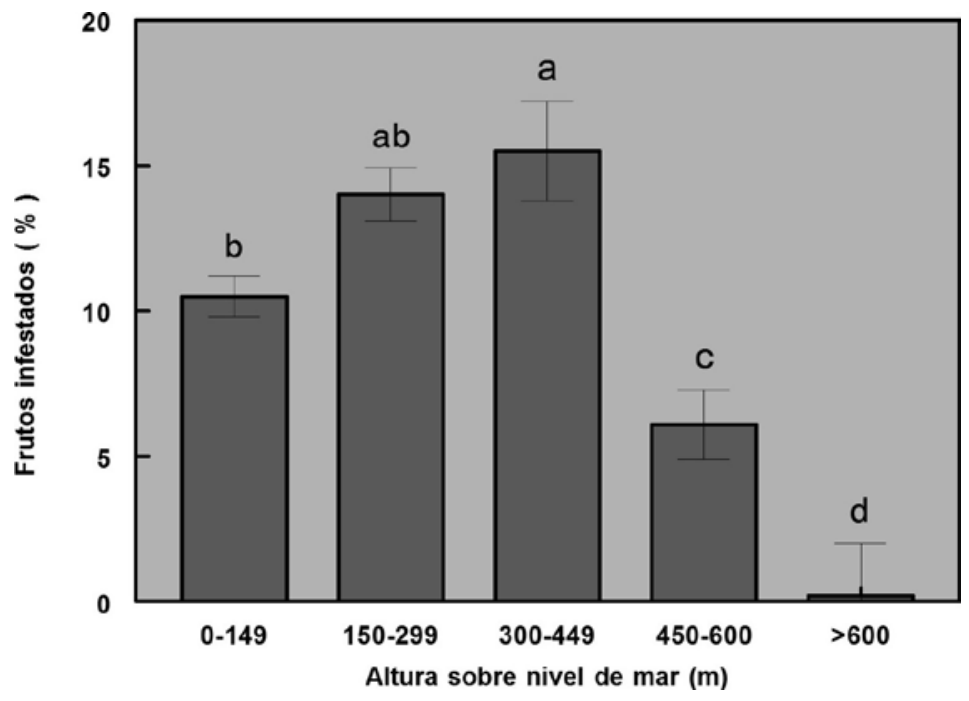

Figura 5. Infestación de frutos de mango por A. tubercularis a diferentes alturas de Nayarit en 2008.

En el último periodo, 'Ataulfo' volvió a registrar los promedios más altos de hembras (4.1), colonias (0.78) y total de escamas (4.9), comparado con 'Tommy Atkins'. Esta misma variedad, también registró la mayor proporción de frutos infestados que 'Tommy Atkins' (Cuadro 6). 
García-Álvarez et al.: Distribución de la escama blanca del mango en Nayarit

Cuadro 6. Densidad de A. tubercularis ( \pm e e) en follaje e infestación de frutos por variedades de mango en Nayarit durante tres años.

\begin{tabular}{lrrrl}
\hline Cultivar & Hembras/hoja & Colonias/hoja & Total/hoja & Frutos infestados (\%) \\
\hline & & 2007 \\
'Ataulfo' & $2.65(0.05) \mathrm{a}$ & $0.80(0.02) \mathrm{a}$ & $3.45(0.1) \mathrm{a}$ & $16.1(0.6) \mathrm{a}$ \\
‘Tommy Atkins' & $1.41(0.06) \mathrm{b}$ & $0.49(0.02) \mathrm{b}$ & $1.90(0.1) \mathrm{b}$ & $12.7(0.7) \mathrm{b}$ \\
& & 2008 & & \\
& $2.95(0.05) \mathrm{a}$ & $1.06(0.02) \mathrm{a}$ & $4.01(0.07) \mathrm{a}$ & $8.6(0.4) \mathrm{a}$ \\
'Ataulfo' & $1.27(0.07) \mathrm{b}$ & $0.58(0.02) \mathrm{b}$ & $1.85(0.08) \mathrm{b}$ & $6.1(0.6) \mathrm{b}$ \\
'Tommy Atkins' & 2009 & & \\
& $4.1(0.1) \mathrm{a}$ & $0.78(0.03) \mathrm{a}$ & $4.9(0.1) \mathrm{a}$ & $35.2(1.6) \mathrm{a}$ \\
'Ataulfo' & $2.2(0.1) \mathrm{a}$ & $0.34(0.03) \mathrm{b}$ & $2.6(0.1) \mathrm{a}$ & $30.6(1.6) \mathrm{b}$ \\
\hline
\end{tabular}

Valores por columna y año con la misma letra son estadísticamente iguales (Tukey, $p \leq 0.05$ ).

Distribución por edad de los huertos. En 2007, la cantidad de hembras, colonias, total de escamas y cantidad de frutos infestados, fueron estadísticamente más altos en huertos de menos de 10 años, que aquellos que tenían más de 10 años (Cuadro 7). En 2008 sucedió lo mismo, la mayor cantidad de hembras (2.77/hoja), colonias (1.05/hoja), total de hembras más colonias (3.82/hoja) e infestación de frutos $(9.5 \%)$, ocurrió en los huertos con menos de 10 años de edad. Finalmente, en el último año también se registraron los promedios más altos (por lo menos el doble) de hembras, colonias y total (hembras+colonias), en huertos de menos de 10 años. Sin embargo, a diferencia de los años anteriores, los huertos más viejos registraron 13\% más infestación de frutos que los más jóvenes (Cuadro 7).

Cuadro 7. Densidad de A. tubercularis en follaje e infestación de frutos de mango ( \pm e e $)$ por variedad en Nayarit durante tres años.

\begin{tabular}{lcrrl}
\hline Edad & Hembras/hoja & Colonias/hoja & Total/hoja & Frutos infestados (\%) \\
\hline & & 2007 \\
$<10$ años & $2.63(0.1) \mathrm{a}$ & $0.87(0.03) \mathrm{a}$ & $3.50(0.11) \mathrm{a}$ & $19.4(0.1) \mathrm{a}$ \\
$>10$ Años & $1.96(0.1) \mathrm{b}$ & $0.61(0.01) \mathrm{b}$ & $2.57(0.06) \mathrm{b}$ & $13.5(0.5) \mathrm{b}$ \\
& & 2008 & & \\
$<10$ Años & $2.77(0.05) \mathrm{a}$ & $1.05(0.02) \mathrm{a}$ & $3.82(0.07) \mathrm{a}$ & $9.5(0.4) \mathrm{b}$ \\
$>10$ años & $1.53(0.07) \mathrm{b}$ & $0.58(0.03) \mathrm{b}$ & $2.10(0.10) \mathrm{b}$ & $4.5(0.5) \mathrm{a}$ \\
& & 2009 & & \\
$<10$ Años & $3.3(0.1) \mathrm{a}$ & $0.63(0.02) \mathrm{a}$ & $3.63(0.1) \mathrm{a}$ & $22.9(2.3) \mathrm{b}$ \\
$>10$ años & $2.6(0.1) \mathrm{b}$ & $0.35(0.04) \mathrm{b}$ & $2.95(0.2) \mathrm{b}$ & $35.9(1.3) \mathrm{a}$ \\
\hline
\end{tabular}

Valores por columna y año con la misma letra son estadísticamente iguales (Tukey, $p \leq 0.05$ ). 
Las observaciones realizadas indican que los bajos niveles de infestación de frutos en 2008 se debieron probablemente a la presencia de "mango niño" (fruto partenocárpico), que en apariencia fue mayor que otros años, aunque esta variable no fue evaluada. Por otra parte, las diferencias detectadas entre los dos cultivares, probablemente se deba a que, en términos generales, los huertos de 'Tommy Atkins' son normalmente más viejos que los de 'Ataulfo' y presentaron ramas viejas con poco rebrote y con follaje escaso, lo cual no favorecería el desarrollo óptimo de la escama. Además, con esta condición se podría genera una menor cantidad de alimento para la escama y una mayor ventilación interna en el dosel, eliminando así la humedad relativa necesaria para el desarrollo de los insectos. Con esta condición de los árboles, además de disponer la escama de menor cantidad de alimento, existe mayor ventilación interna en el dosel lo que no genera una humedad relativa adecuada para el desarrollo de las escamas. Por otra parte, la infestación de frutos fue consistente con la infestación en follaje, ya que tanto en 'Ataulfo' como en huertos de menos de 10 años de edad ocurrieron poblaciones más altas en el follaje y en los frutos (Cuadros 6 y 7).

\section{CONCLUSIONES}

Los huertos de mango localizados en poblaciones al sur del estado, presentaron las mayores densidades de escama blanca en el follaje, así como las mayores infestaciones de frutos.

Los municipios de Bahía de Banderas y de Compostela y registraron las mayores infestaciones de escama blanca en hojas y frutos; San Blas y Tepic presentaron infestaciones moderadamente altas; mientras que Santiago Ixcuintla y Tecuala tuvieron las infestaciones más bajas. Hasta el término del estudio, no se detectó la escama en huertos de Acaponeta, Rosamorada y Ruiz.

Las mayores infestaciones de la escama blanca del mango se encontraron en huertos situados en alturas de $0-500 \mathrm{msnm}$; y de moderadas a muy bajas, en huertos de 500-800 metros sobre el nivel del mar.

Las poblaciones más grandes de la escama blanca del mango y las de mayor infestación de frutos se registraron en la variedad 'Ataulfo', así como en huertos menores de 10 años de edad.

AGRADECIMIENTOS. Esta investigación fue financiada por el Fondo Sectorial SAGARPA-CONACYT, proyectos 2006-48122 y 2011-12-171759.

\section{LITERATURA CITADA}

Arias, de L. M., Jines C., A., Carrera, C., Bustos, N., Plúas M., P. \& Gutiérrez, K. 2004. Biología, dinámica poblacional, muestreo, nivel de daño y alternativas para el manejo de Aulacaspis tubercularis en mango de exportación. Instituto Nacional Autónomo de Investigaciones Agropecuarias, Folleto Técnico No. 56. Guayaquil, Ecuador 19 pp. 
González-Carrillo, J. A., Urías-López, M. A. \& García-Álvarez, N. C. 2008a. Fluctuación poblacional de la escama blanca, Aulacaspis tubercularis Newstead (Hemiptera: Diaspididae) del mango en Nayarit, México. Entomología Mexicana, 7: 646-651.

González-Carrillo J. A., Urías-López M. A. \& García-Álvarez N. C. 2008b. Fluctuación poblacional de insectos depredadores de la escama blanca (Hemiptera: Diaspididae) del mango en Nayarit. Memoria del XXXI Congreso. Zacatecas, Zac. Nacional de Control Biológico. Sociedad Mexicana de Control Biológico.

Le Lagadec M. D., Louw, C. E. \& Labushagne, C. 2006. The control of scale insects and mealybugs on mangoes in South Africa using neo-nicotenoids: A review of experimental work from 2001 to 2005. Acta Horticulturae, 820: 549-557.

SAGARPA-CESAVENAY. 2007. Superficie de mango, volumen de producción y rendimiento promedio por superficie y por variedad. Subdirección Agropecuaria. Programa de Fomento Agrícola y Programa de Sanidad Vegetal. Delegación Estatal de Nayarit. 10 pp.

SAS Institute. 2000. SAS/STAT user's guide. Version 8. SAS Institute Inc. Carey, N.C.

Triplehorn, C. A. \& Johnson, N. F. 2005. Borror and Delongs' introduction to the study of insects. Seventh edition. Brooks/Cole. Belmont CA, U.S.A. 864 p.

Urías, L., M. A. 2006. Principales plagas del mango en Nayarit, pp. 211-234. In: V. Vázquez V. \& M. H. Pérez B. (Eds.). El cultivo del mango: Principios y tecnología de producción. Instituto Nacional de Investigaciones Forestales Agrícolas y Pecuarias. Santiago Ixcuintla, Nay.

Urías-López, M. A. \& Flores-Canales, R. 2005. La "escama blanca", Aulacaspis tubercularis Newstead (Homoptera: Diaspididae) una nueva plaga del mango: Fluctuación poblacional y anotaciones biológicas. Entomología Mexicana, 4: 579-584.

Urías-López, M. A., Hernández-Fuentes, L. M., Osuna-García, J. A., Pérez-Barraza, M. H., García-Álvarez, N. C. \& González-Carrillo, J. A. 2013. Aspersiones de insecticidas en campo sobre la escama blanca del mango (Hemiptera: Diaspididae). Revista Fitotecnia Mexicana, 36: 173-180

Urías-López, M. A., Osuna-García, J. A., Vázquez-Valdivia, V. \& Pérez-Barraza, M. H. 2010. Fluctuación poblacional y distribución de la escama blanca del mango (Aulacaspis tubercularis Newstead) en Nayarit, México. Revista Chapingo Serie Horticultura, 16: 77-82.

Wysoki, M., Ben-Dov, Y., Swirski, E. \& Izhar, Y. 1993. The arthropod pests of mango in Israel. Acta Horticulturae, 341: 452-466. 